Algebraic $\&$ Geometric $\mathcal{T}_{\text {opology }}$

Volume 4 (2004) 781-812

Published: 23 September 2004

ATG

\title{
Duality and Pro-Spectra
}

\author{
J. Daniel Christensen \\ DANIEL C. ISAKSEN
}

\begin{abstract}
Cofiltered diagrams of spectra, also called pro-spectra, have arisen in diverse areas, and to date have been treated in an ad hoc manner. The purpose of this paper is to systematically develop a homotopy theory of pro-spectra and to study its relation to the usual homotopy theory of spectra, as a foundation for future applications. The surprising result we find is that our homotopy theory of pro-spectra is Quillen equivalent to the opposite of the homotopy theory of spectra. This provides a convenient duality theory for all spectra, extending the classical notion of Spanier-Whitehead duality which works well only for finite spectra. Roughly speaking, the new duality functor takes a spectrum to the cofiltered diagram of the SpanierWhitehead duals of its finite subcomplexes. In the other direction, the duality functor takes a cofiltered diagram of spectra to the filtered colimit of the Spanier-Whitehead duals of the spectra in the diagram. We prove the equivalence of homotopy theories by showing that both are equivalent to the category of ind-spectra (filtered diagrams of spectra). To construct our new homotopy theories, we prove a general existence theorem for colocalization model structures generalizing known results for cofibrantly generated model categories.
\end{abstract}

AMS Classification 55P42; 55P25, 18G55, 55U35, 55Q55

Keywords Spectrum, pro-spectrum, Spanier-Whitehead duality, closed model category, colocalization

\section{Introduction}

In recent years there have been many areas in which cofiltered diagrams of spectra have naturally arisen as a way to organize homotopical information. For example, see the work of Cohen, Jones and Segal [7] and Hurtubise [15] on Floer homology theory, Ando and Morava 1] on formal groups and free loop spaces, and unpublished work of Dwyer and Rezk and of Arone on Goodwillie calculus. Pro-spectra are also likely to be the target of an étale realization functor on stable motivic homotopy theory [18]. 
A cofiltered diagram is called a pro-spectrum (see Section 3) and in general it contains more information than its homotopy limit. Thus it is necessary to develop a homotopy theory of pro-spectra, and our goal is to do this systematically and to study its relation to the usual homotopy theory of spectra. We expect that this framework will be useful for many applications.

Spanier-Whitehead duality was one of the reasons for which the stable homotopy category was invented [5] 21] 24]. The idea is that there is a contravariant functor from the stable homotopy category to itself that induces an equivalence between the homotopy category of finite spectra and its own opposite. This functor is defined by taking a spectrum $X$ to the function spectrum $F\left(X, S^{0}\right)$, where $S^{0}$ is the sphere spectrum.

One important property of Spanier-Whitehead duality is that the double dual of an infinite spectrum is not weakly equivalent to the original spectrum. In some contexts, this is a useful property because it gives a method for producing new interesting spectra. On the other hand, it is sometimes inconvenient that Spanier-Whitehead duality does not give an equivalence between the whole stable homotopy category and its opposite.

The same situation arises in linear algebra over a field $k$. The functional dual induces an equivalence of the category of finite dimensional $k$-vector spaces with its own opposite, but it does not extend to an equivalence on the whole category of $k$-vector spaces. One solution is to pass to the category of pro-finite $k$-vector spaces. In fact, the category of $k$-vector spaces is equivalent to the opposite of the category of pro-finite $k$-vector spaces.

Because of the strong analogy between the stable homotopy category and categories of chain complexes, it is natural to ask whether the same kind of solution works for spectra. The main result of this paper is that it does. We define a homotopy theory for the category of pro-spectra and show that its opposite is equivalent to the usual homotopy theory of spectra. One use of this description of the opposite of stable homotopy theory is to work in progress on a classification of polynomial functors (in the sense of Goodwillie calculus) from spaces to spectra [2].

The situation for pro-spectra is significantly more complicated than for provector spaces because of the intricacies of homotopy categories. The most naive approach is to consider pro-objects in the homotopy category of spectra. The unstable version of this approach appears in [4] and 25]. Homotopy theorists have learned through countless examples that considering diagrams in a homotopy category is usually the wrong viewpoint. Rather, it is better to consider commutative diagrams in a geometric category and then study the 
homotopy theory of these diagrams. Following this philosophy, we consider the category of pro-objects in a geometric category of spectra and then equip it with a homotopy theory.

More precisely, we construct a model structure on pro-spectra in which the weak equivalences are detected by cohomotopy groups. This model structure is contravariantly Quillen equivalent to a model structure on the category of ind-spectra (i.e., the category of filtered systems of spectra) in which the weak equivalences are detected by homotopy groups. The model structure on indspectra is in turn Quillen equivalent to the usual stable model structure for spectra.

The cofibrant pro-spectra in our new model structure are easy to describe. They are the pro-spectra that are essentially levelwise cofibrant, that is, they are levelwise cofibrant up to isomorphism. The fibrant pro-spectra are only slightly harder to describe. They are the strictly fibrant pro-spectra that are essentially levelwise homotopy-finite, that is, the strictly fibrant pro-spectra (see Section 3.4) that are also levelwise weakly equivalent to a finite complex, up to isomorphism. Dually, the fibrant ind-spectra are the essentially levelwise fibrant ind-spectra, and the cofibrant ind-spectra are the strictly cofibrant indspectra that are essentially levelwise homotopy-finite. The importance of finite complexes is no surprise since we are defining homotopy theories that work well with respect to Spanier-Whitehead duality.

The description of fibrant pro-spectra in terms of homotopy-finite spectra allows us to compute the total derived functor Rlim of the limit functor from prospectra to spectra. For a constant pro-spectrum $X, \operatorname{Rlim} X$ is the SpanierWhitehead double dual of $X$. See Remark 6.9 for more details.

Our chief tool for establishing the appropriate homotopy theories of pro-spectra and ind-spectra is a general existence theorem for a certain kind of colocalization of model categories (see Theorem [2.6). This result is a generalization of [12, Thm. 5.1.1] because cofibrantly generated model structures satisfy our hypotheses, and our proof is very similar. In our application, we begin with the strict model structure on pro-spectra in which the weak equivalences are, up to isomorphism, the levelwise weak equivalences (see Section 3.4). Then we use mapping spaces into the spheres to determine the local weak equivalences of pro-spectra; note that the model structure on pro-spectra is a localization, not a colocalization, because we use the dual of Theorem 2.6. Dually, for indspectra, we start with the strict structure and then use mapping spaces out of the spheres to determine the colocal weak equivalences. 
In this paper, we need a model for spectra that has a well-behaved function spectrum defined on the geometric category, not just on the homotopy category. We use symmetric spectra [14 for this model. Section 5 reviews the relevant ideas. It is also possible to work entirely in the category of $S$-modules [11].

In fact, we do not really need the full power of a general function spectrum construction. Rather, we only need to define function spectra of the form $F\left(X, S^{0}\right)$. It has been suggested to us that this is probably possible on more naive categories of spectra such as the one described in [6], but we have not checked the details.

It is important that the techniques that we develop here can be applied to other situations involving pro-spectra. For example, if $E$ is any generalized cohomology theory, then we can define a model structure on the category of prospectra in which the weak equivalences are detected by $E$-cohomology groups. And the proof of our duality result extends to a proof that this model category is Quillen equivalent to the opposite of the model category of $\operatorname{End}(E)$-module spectra. Thus, we have essentially constructed all cohomological localizations for pro-spectra. This is relevant to work in progress involving étale homotopy types and quadratic forms over arbitrary fields [8]. This kind of situation also occurs in 20].

We assume that the reader is familiar with the language and basic results of model categories. The original reference is [23], but we conform to the notations and terminology of [12. See also [9] or [13].

\section{$1.1 \quad$ Organization}

The paper is organized as follows. We begin with the general existence theorem for $K$-colocal model structures. Next we review the theory of pro-categories and ind-categories. Then we apply our colocalization result to pro-categories and ind-categories.

The second part of the paper begins with a review of some details about symmetric spectra. Next we construct and study the model structures for prospectra and ind-spectra. Finally, we prove that the various model structures are Quillen equivalent.

\subsection{Acknowledgements}

The authors thank the SFB 343 at Universität Bielefeld, Germany. They also thank Greg Arone for originally motivating the project and Stefan Schwede for 
useful conversations. The first author was supported by an NSERC Research Grant and the second author was supported by an NSF Postdoctoral Research Fellowship.

\section{Colocalizations of Model Structures}

In this section, we prove a general theorem about colocalization of model structures. Much of what appears here is very similar to [12, Ch. 5]. One important difference is that we work with model structures that may not be cofibrantly generated.

Start with a right proper model category $\mathcal{C}$, that is, a model category in which the pullback of a weak equivalence along a fibration is always a weak equivalence. We refer to the cofibrations, weak equivalences, and fibrations of $\mathcal{C}$ as underlying cofibrations, weak equivalences, and fibrations. For convenience, assume that $\mathcal{C}$ is simplicial and write $\operatorname{Map}(\cdot, \cdot)$ for the simplicial mapping space. In fact, the results of this section carry over to the non-simplicial setting, but one must use the technical machinery of homotopy function complexes 12 Ch. 17].

Let $K$ be a set of objects of $\mathcal{C}$. Since we shall only use the homotopical properties of the objects in $K$, we may as well assume that each object in $K$ is underlying cofibrant.

Definition 2.1 A map $f: X \rightarrow Y$ in $\mathcal{C}$ is a $K$-colocal weak equivalence if for each $A$ in $K$, the map

$$
\operatorname{Map}(A, \hat{X}) \rightarrow \operatorname{Map}(A, \hat{Y})
$$

is a weak equivalence of simplicial sets, where $\hat{X} \rightarrow \hat{Y}$ is a fibrant replacement for $X \rightarrow Y$, that is, there is a commuting square

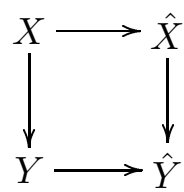

whose rows are underlying fibrant replacements.

The idea is that we detect $K$-colocal weak equivalences by considering maps out of objects in $K$. 
Observe that the choice of fibrant replacements for $X$ and $Y$ does not matter; if the map

$$
\operatorname{Map}(A, \hat{X}) \rightarrow \operatorname{Map}(A, \hat{Y})
$$

is a weak equivalence for one choice of fibrant replacements, then it is a weak equivalence for any other choice of fibrant replacements. Also note that underlying weak equivalences are automatically $K$-colocal weak equivalences.

Definition 2.2 A map in $\mathcal{C}$ is a $K$-colocal fibration if it is an underlying fibration. A map in $\mathcal{C}$ is a $K$-colocal cofibration if it has the left lifting property with respect to all $K$-colocal acyclic fibrations.

Because there is no difference between underlying fibrations and $K$-colocal fibrations, we use the term "fibration" unambiguously for maps in either class.

We are defining a right Bousfield localization of the model category $\mathcal{C}$ in the sense of [12, Defn. 3.3.1]. We add more weak equivalences, keep the fibrations unchanged, and define the cofibrations to be what they must be.

Theorem 2.6] states that under some general hypotheses on $\mathcal{C}$, our definitions are a model structure. However, the two-out-of-three axiom and the retract axiom are satisfied in general. This follows from an inspection of the definitions.

The following results basically appear in [12, Ch. 5] with minor obvious changes in the proofs.

\section{Lemma 2.3}

(a) The class of $K$-colocal acyclic cofibrations is the same as the class of underlying acyclic cofibrations.

(b) Let $A$ be any object of $K$. For $n \geq 0$, the map $i: \partial \Delta[n] \otimes A \rightarrow \Delta[n] \otimes A$ is a $K$-colocal cofibration.

(c) Let $p: X \rightarrow Y$ be a fibration between fibrant objects $X$ and $Y$. Then $p$ is a $K$-colocal acyclic fibration if and only if it has the right lifting property with respect to every underlying cofibration $\partial \Delta[n] \otimes A \rightarrow \Delta[n] \otimes A$ in which $A$ belongs to $K$ and $n \geq 0$.

Proof For part (a), the proof of [12, Lem. 5.3.2] works word for word. For parts (b) and (c), the proofs of [12, Prop. 5.2.5] and [12, Prop. 5.2.4] also work. Although we do not have a set of generating acyclic cofibrations at our disposal, we have avoided this necessity by assuming that the map in part (c) is already a fibration. 
In order to prove the rest of the model structure axioms, we must add hypotheses on $\mathcal{C}$.

Hypothesis 2.4 Let $\mathcal{C}$ be a right proper simplicial model category, and let $K$ be a set of cofibrant objects in $\mathcal{C}$. Suppose that there exists a regular cardinal $\kappa$ with the following properties. First, each object of $K$ is $\kappa$-small relative to the underlying cofibrations. Second, if

$$
X_{0} \rightarrow X_{1} \rightarrow \cdots \rightarrow X_{\beta} \rightarrow \cdots
$$

is a $\kappa$-sequence of underlying cofibrations and $p: \operatorname{colim}_{\beta} X_{\beta} \rightarrow Y$ is a map such that the composition $p_{\beta}: X_{\beta} \rightarrow Y$ is a fibration for each successor ordinal $\beta$, then $p$ is also a fibration.

See Section 3.3 for a review of the notions of smallness and $\kappa$-sequences.

The idea is that fibrations are closed under a certain kind of sufficiently long sequential colimit. If $\mathcal{C}$ is cofibrantly generated, then we may choose $\kappa$ such that the domains of each of the underlying generating acyclic cofibrations as well as the objects of $K$ are $\kappa$-small relative to the underlying cofibrations. Hence cofibrantly generated model categories always satisfy Hypothesis 2.4. However, in our intended application to pro-spectra, $\mathcal{C}$ is not cofibrantly generated, but the above hypothesis is still satisfied.

It is not usually a problem to find a single $\kappa$ for which each $A$ in $K$ is $\kappa$-small. As long as each $A$ is $\kappa_{A}$-small for some $\kappa_{A}$, we may choose $\kappa$ to be an upper bound for the ordinals $\kappa_{A}$.

The following lemma is not a factorization axiom for the $K$-colocal model structure that we are constructing. The problem is that $K$-colocal acyclic fibrations are not detected by the right lifting property with respect to the maps $\partial \Delta[n] \otimes A \rightarrow \Delta[n] \otimes A$. See [12, Ex. 5.2.7] for an example of this problem. Using this lemma, the factorization we want follows from [12, Prop. 5.3.5].

Lemma 2.5 Under Hypothesis 2.4, every map $f: X \rightarrow Y$ has a factorization into a $K$-colocal cofibration $i: X \rightarrow W$ followed by a fibration $p: W \rightarrow Y$ that has the right lifting property with respect to all maps $\partial \Delta[n] \otimes A \rightarrow \Delta[n] \otimes A$ for $A$ in $K$.

Proof We use a variation on the small object argument [12, $\S 10.5]$. Let $J_{0}$ be the set of all squares

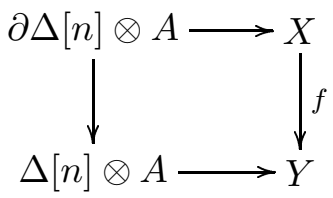

Algebraic 83 Geometric Topology, Volume 4 (2004) 
for which $A$ belongs to $K$. Define $Z_{0}$ to be the pushout

$$
\left(\coprod_{J_{0}} \Delta[n] \otimes A\right) \coprod_{J_{0}} \coprod_{\partial \Delta[n] \otimes A} X,
$$

and let $j_{0}: X \rightarrow Z_{0}$ and $q_{0}: Z_{0} \rightarrow Y$ be the obvious maps.

Now factor the map $q_{0}$ into an underlying acyclic cofibration $i_{0}: Z_{0} \rightarrow W_{0}$ followed by a fibration $p_{0}: W_{0} \rightarrow Y$. This finishes the first stage of the factorization.

We build the whole factorization by a transfinite induction of length $\kappa$. If $\beta$ is a limit ordinal, then set $W_{\beta}$ to be $\operatorname{colim}_{\alpha<\beta} W_{\alpha}$ and set $p_{\beta}$ to be $\operatorname{colim}_{\alpha<\beta} p_{\alpha}$.

On the other hand, if $\beta$ is a successor ordinal, then define $J_{\beta}$ to be the set of all squares

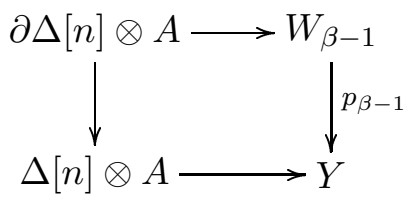

for which $A$ belongs to $K$. Define $Z_{\beta}$ to be the pushout

$$
\left(\coprod_{J_{\beta}} \Delta[n] \otimes A\right) \coprod_{J_{\mathcal{\beta}}} \coprod_{\partial \Delta[n] \otimes A} W_{\beta-1},
$$

and let $j_{\beta}: X \rightarrow Z_{\beta}$ and $q_{\beta}: Z_{\beta} \rightarrow Y$ be the obvious maps. Now factor the map $q_{\beta}$ into an underlying acyclic cofibration $i_{\beta}: Z_{\beta} \rightarrow W_{\beta}$ followed by an underlying fibration $p_{\beta}: W_{\beta} \rightarrow Y$. This finishes the $\beta$ th stage of the factorization.

Transfinite induction yields a $\kappa$-sequence

$$
X \rightarrow W_{0} \rightarrow W_{1} \rightarrow \cdots \rightarrow W_{\beta} \rightarrow \cdots .
$$

Note that there are compatible maps $p_{\beta}: W_{\beta} \rightarrow Y$ for every $\beta$. Let $W$ be $\operatorname{colim}_{\beta} W_{\beta}$, and let $p: W \rightarrow Y$ be $\operatorname{colim}_{\beta} p_{\beta}$. By construction, the maps $p_{\beta}$ are fibrations for every successor ordinal $\beta$. Hypothesis 2.4 implies that $p$ is a fibration. Observe that $p$ has the right lifting property with respect to the maps $\partial \Delta[n] \otimes A \rightarrow \Delta[n] \otimes A$ as in the usual small object argument because each $A$ is $\kappa$-small.

It remains to show that the map $i: X \rightarrow W$ is a $K$-colocal cofibration. Since $K$-colocal cofibrations are defined by a left lifting property and since $i$ is a 
transfinite composition of the maps $W_{\beta} \rightarrow W_{\beta+1}$, it suffices to show that each $W_{\beta} \rightarrow W_{\beta+1}$ is a $K$-colocal cofibration. But this map is the composition of $W_{\beta} \rightarrow Z_{\beta+1}$ and $Z_{\beta+1} \rightarrow W_{\beta+1}$. The second map is an underlying acyclic cofibration by construction, so it is a $K$-colocal cofibration by Lemma [2.3)(a). The first map is a cobase change of a coproduct of maps of the form $\partial \Delta[n] \otimes A \rightarrow$ $\Delta[n] \otimes A$, each of which is a $K$-colocal cofibration by Lemma 2.3(b). Therefore, $W_{\beta} \rightarrow Z_{\beta+1}$ is also a $K$-colocal cofibration.

Theorem 2.6 Under Hypothesis 2.4, the $K$-colocal cofibrations, $K$-colocal weak equivalences, and fibrations of Definitions 2.1] and 2.2 give a right proper simplicial $K$-colocal model structure on $\mathcal{C}$.

Remark 2.7 The assumption that $\mathcal{C}$ be simplicial is not essential. In fact, the theorem is true when $\mathcal{C}$ is not simplicial (except that the $K$-colocal model structure is also not simplicial). The proof is similar in spirit but uses the technical machinery of homotopy function complexes [12, Ch. 17].

Proof From the basic axioms, only the lifting and factorization axioms require explanation. One half of the lifting axiom follows from the definitions. The other half of the lifting axiom and one half of the factorization axiom follow from the fact that $K$-colocal fibrations are the same as underlying fibrations and that $K$-colocal acyclic cofibrations are the same as underlying acyclic cofibrations (see Lemma 2.3(a)). It remains only to factor maps into $K$-colocal cofibrations followed by $K$-colocal acyclic fibrations. These factorizations can be constructed as in [12, Prop. 5.3.5] (see also [12, Prop. 5.3.4]) using Lemma 2.5

This finishes the basic model structure axioms. Right properness follows immediately from [12, Prop. 3.4.4]. The simplicial structure can be deduced from [12, $\S 5.4 .4]$. Tensors, cotensors, and mapping spaces are defined as in the underlying model structure.

When we consider pro-categories later, we will actually apply the dual of Theorem 2.6] to obtain localizations rather than colocalizations.

\section{Preliminaries on Pro-Categories}

We now establish some background on pro-categories and ind-categories. Whenever possible, we use dual definitions and arguments. 


\subsection{Pro-Categories and Ind-Categories}

Definition 3.1 For a category $\mathcal{C}$, the category pro- $\mathcal{C}$ has objects all cofiltered diagrams in $\mathcal{C}$, and

$$
\operatorname{Hom}_{\text {pro-C }}(X, Y)=\lim _{s} \operatorname{colim}_{t} \operatorname{Hom}_{\mathcal{C}}\left(X_{t}, Y_{s}\right) \text {. }
$$

Composition is defined in the natural way.

A category $I$ is cofiltered if the following conditions hold: it is non-empty and small; for every pair of objects $s$ and $t$ in $I$, there exists an object $u$ together with maps $u \rightarrow s$ and $u \rightarrow t$; and for every pair of morphisms $f$ and $g$ with the same source and target, there exists a morphism $h$ such that $f h$ equals $g h$. Recall that a category is small if it has only a set of morphisms. A diagram is said to be cofiltered if its indexing category is so.

Objects of pro- $\mathcal{C}$ are functors from cofiltered categories to $\mathcal{C}$. The original references for pro-categories are [3] and [4].

Definition 3.2 For a category $\mathcal{C}$, the category ind- $\mathcal{C}$ has objects all filtered diagrams in $\mathcal{C}$, and

$$
\operatorname{Hom}_{\text {ind- } \mathcal{C}}(X, Y)=\lim _{t} \operatorname{colim}_{s} \operatorname{Hom}_{\mathcal{C}}\left(X_{t}, Y_{s}\right) .
$$

Composition is defined in the natural way.

The notion of a filtered diagram is dual to that of a cofiltered diagram. Namely, for every pair of objects $s$ and $t$ in the indexing category, there exists an object $u$ together with maps $s \rightarrow u$ and $t \rightarrow u$; for every pair of morphisms $f$ and $g$ in the indexing category with the same source and target, there exists a morphism $h$ such that $h f$ equals $h g$; and the category is small and non-empty.

Objects of ind- $\mathcal{C}$ are functors from filtered categories to $\mathcal{C}$. See 3$]$ or $[10$ for more background on the definition of ind-categories. For every category $\mathcal{C}$, the category (pro- $\mathcal{C})^{\mathrm{op}}$ is isomorphic to the category ind- $\left(\mathcal{C}^{\mathrm{op}}\right)$. Therefore, ind-categories can be studied by considering pro-categories and appealing to duality.

The words "pro-object" and "ind-object" refer to objects of pro-categories and ind-categories respectively. A constant pro-object or ind-object is (isomorphic to) one indexed by the category with one object and one identity map. Let $c: \mathcal{C} \rightarrow$ pro- $\mathcal{C}$ be the functor taking an object $X$ to the constant pro-object with value $X$. Similarly, let $c: \mathcal{C} \rightarrow$ ind- $\mathcal{C}$ take an object to its associated constant ind-object. Note that these functors make $\mathcal{C}$ a full subcategory of both pro- $\mathcal{C}$ and ind- $\mathcal{C}$. 


\subsection{Level Representations}

A level representation of a map of pro-objects $f: X \rightarrow Y$ is: a cofiltered index category $I$; pro-objects $\tilde{X}$ and $\tilde{Y}$ indexed by $I$; pro-isomorphisms $X \rightarrow \tilde{X}$ and $Y \rightarrow \tilde{Y}$; and a collection of maps $f_{s}: \tilde{X}_{s} \rightarrow \tilde{Y}_{s}$ for all $s$ in $I$ such that for all $t \rightarrow s$ in $I$, there is a commutative diagram

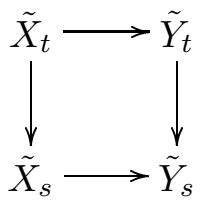

and such that the maps $f_{s}$ represent a pro-map $\tilde{f}: \tilde{X} \rightarrow \tilde{Y}$ belonging to a commutative square

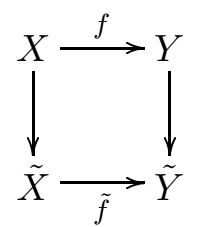

in pro- $\mathcal{C}$. In other words, a level representation of a pro-map $f$ is just a natural transformation such that the maps $f_{s}$ represent the element $f$ of

$$
\lim _{s} \operatorname{colim}_{t} \operatorname{Hom}_{\mathcal{C}}\left(X_{t}, Y_{s}\right) \cong \lim _{s} \operatorname{colim}_{t} \operatorname{Hom}_{\mathcal{C}}\left(\tilde{X}_{t}, \tilde{Y}_{s}\right) .
$$

A similar definition applies to level representations of ind-maps. Every map in pro- $\mathcal{C}$ (and in ind- $\mathcal{C}$ ) has a level representation [4, Appendix 3.2] [22].

A pro-object (resp., ind-object) $X$ satisfies a certain property levelwise if each $X_{s}$ satisfies that property. Similarly, a level representation $X \rightarrow Y$ satisfies a certain property levelwise if each $X_{s} \rightarrow Y_{s}$ satisfies that property. A proobject, ind-object or map satisfies a certain property essentially levelwise if up to isomorphism it satisfies that property levelwise.

\subsection{Smallness and Cosmallness}

In this section we provide some results about small objects in ind-categories and cosmall objects in pro-categories.

Let $\lambda$ be any ordinal. Then $\lambda$ is the partially ordered set of all ordinals strictly less than $\lambda$. A $\lambda$-sequence $X$ in a category $\mathcal{C}$ is a functor from $\lambda$ to $\mathcal{C}$ such 
that the natural map $\operatorname{colim}_{\beta<\alpha} X_{\beta} \rightarrow X_{\alpha}$ is an isomorphism for every limit ordinal $\alpha<\lambda$. In other words, it is a diagram

$$
X_{0} \rightarrow X_{1} \rightarrow \cdots \rightarrow X_{\beta} \rightarrow \cdots
$$

of length $\lambda$ with a kind of continuity condition at the limit ordinals. Note that $X_{\beta}$ is defined only for $\beta<\lambda$, not for $\beta=\lambda$. A $\lambda$-tower is a contravariant functor from $\lambda$ to $\mathcal{C}$ such that the natural map $X_{\alpha} \rightarrow \lim _{\beta<\alpha} X_{\beta}$ is an isomorphism for every limit ordinal $\alpha<\lambda$. In other words, it is a diagram

$$
\cdots \rightarrow X_{\beta} \rightarrow \cdots \rightarrow X_{1} \rightarrow X_{0}
$$

with a kind of continuity condition at the limit ordinals.

Recall that a cardinal $\lambda$ is regular if the disjoint union

$$
\coprod_{\beta \in \mu} \nu_{\beta}
$$

is smaller than $\lambda$ whenever $\mu$ is a cardinal smaller than $\lambda$ and $\nu_{\beta}$ is a cardinal smaller than $\lambda$ for every $\beta$ in $\mu$. The relevance of regular cardinals is that a transfinite sequence of maps whose length is a regular cardinal has no cofinal subsequence of shorter length.

Recall that an object $X$ of a category $\mathcal{C}$ is $\kappa$-small relative to a class of maps $C$ [12, Defn. 10.4.1] if for every regular cardinal $\lambda \geq \kappa$ and every $\lambda$-sequence $Y$ such that each $Y_{\beta} \rightarrow Y_{\beta+1}$ belongs to $C$, the natural map

$$
\underset{\alpha}{\operatorname{colim}} \operatorname{Hom}\left(X, Y_{\alpha}\right) \rightarrow \operatorname{Hom}\left(X, \operatorname{colim}_{\alpha} Y_{\alpha}\right)
$$

is an isomorphism. The idea is that every map from $X$ to colim $Y$ must factor through some $Y_{\alpha}$, and this factorization is unique up to refinement. Dually, an object $X$ is $\kappa$-cosmall relative to $C$ if for every regular cardinal $\lambda \geq \kappa$ and every $\lambda$-tower $Y$ such that each $Y_{\beta+1} \rightarrow Y_{\beta}$ belongs to $C$, the natural map

$$
\underset{\alpha}{\operatorname{colim}} \operatorname{Hom}\left(Y_{\alpha}, X\right) \rightarrow \operatorname{Hom}\left(\lim _{\alpha} Y_{\alpha}, X\right)
$$

is an isomorphism. Again, the idea is that every map from $\lim Y$ to $X$ must factor through some $Y_{\alpha}$ uniquely up to refinement.

Smallness is a common feature of objects of most familiar set-based categories. For example, every simplicial set is $\kappa$-small relative to all maps for some $\kappa$. On the other hand, cosmallness is a rare property; the only cosmall objects (relative to all maps) in the categories of topological spaces, simplicial sets, sets, groups, sheaves, etc. are the terminal objects! Nonetheless, pro-categories have many cosmall objects. 
Proposition 3.3 Let $\mathcal{C}$ be any category, and let $\omega$ be the first infinite ordinal. Every constant pro-object of pro- $\mathcal{C}$ is $\omega$-cosmall relative to all pro-maps. More generally, if $X$ is a pro-object and $\kappa$ is an infinite regular cardinal such that $X$ can be represented by a diagram with fewer than $\kappa$ morphisms, then $X$ is $\kappa$-cosmall relative to all pro-maps.

Proof Let $X$ be a cofiltered diagram with fewer than $\kappa$ morphisms, and let $Y$ be a $\lambda$-tower in pro- $\mathcal{C}$ for some regular cardinal $\lambda \geq \kappa$. By [17, Thm. 7.2], $\operatorname{Hom}_{\text {pro }}\left(\lim _{\beta} Y^{\beta}, c X_{t}\right)$ is isomorphic to $\operatorname{colim}_{\beta} \operatorname{Hom}_{\text {pro }}\left(Y^{\beta}, c X_{t}\right)$ for every $t$. After taking limits with respect to $t$, we conclude that $\operatorname{Hom}_{\text {pro }}\left(\lim _{\beta} Y^{\beta}, X\right)$ is isomorphic to $\lim _{t} \operatorname{colim}_{\beta} \operatorname{Hom}_{\text {pro }}\left(Y^{\beta}, c X_{t}\right)$. The indexing category for the limit is smaller than $\kappa$, and the indexing category for the sequential colimit is at least as large as $\kappa$. The following lemma tells us that the limit and colimit commute, and therefore $\operatorname{Hom}_{\text {pro }}\left(\lim _{\beta} Y^{\beta}, X\right)$ is isomorphic to $\operatorname{colim}_{\beta} \operatorname{Hom}_{\text {pro }}\left(Y^{\beta}, X\right)$. This shows that $X$ is $\kappa$-cosmall.

Lemma 3.4 Let $I$ be a cofiltering category and $\lambda$ be a regular cardinal such that $I$ has fewer than $\lambda$ morphisms. Suppose that $(t, \beta) \mapsto F_{\beta}^{t}$ is a set-valued functor on $I \times \lambda$. Then the natural map

$$
\underset{\beta \in \lambda}{\operatorname{colim}} \lim _{t \in I} F_{\beta}^{t} \rightarrow \lim _{t \in I} \operatorname{colim}_{\beta \in \lambda} F_{\beta}^{t}
$$

is an isomorphism.

Proof For surjectivity, a typical element of the target is a compatible family $\left\{\overline{f^{t}}\right\}$, where each $\overline{f^{t}}$ belongs to $\operatorname{colim}_{\beta} F_{\beta}^{t}$. Now $\overline{f^{t}}$ is represented by an element $f_{\beta(t)}^{t}$. Since $I$ is smaller than $\lambda$ and $\lambda$ is regular, there exists $\alpha<\lambda$ such that $\beta(t) \leq \alpha$ for all $t$. In other words, each $\overline{f^{t}}$ is represented by an element $f_{\alpha}^{t}$ of $F_{\alpha}^{t}$. These elements do not necessarily give us an element of $\lim _{t} F_{\alpha}^{t}$ because they might not be compatible. However, the original compatibility of the family $\left\{\overline{f^{t}}\right\}$ tells us that for each map $i: t \rightarrow s$ of $I, f_{\alpha}^{t}$ and $f_{\alpha}^{s}$ become compatible after further refinement to some $\alpha(i)$. Again because $I$ is smaller than $\lambda$, there exists a $\gamma$ such that $\gamma<\lambda$ and $\alpha(i) \leq \gamma$ for all maps $i: t \rightarrow s$ of $I$. This shows that the map is surjective.

For injectivity, now suppose given two elements $f$ and $g$ of the source that agree in the target. We may as well assume that they are both represented by elements of $\lim _{t} F_{\beta}^{t}$ for some $\beta$. In other words, we have two compatible families $\left\{f_{\beta}^{t}\right\}$ and $\left\{g_{\beta}^{t}\right\}$, where each $f_{\beta}^{t}$ and $g_{\beta}^{t}$ belongs to $F_{\beta}^{t}$. Since $f$ and $g$ agree in the target, we know that $f_{\beta}^{t}$ and $g_{\beta}^{t}$ represent the same element of $\operatorname{colim}_{\beta} F_{\beta}^{t}$ 
for each $t$. This means that $f_{\beta}^{t}$ and $g_{\beta}^{t}$ become equal after refinement to some $\beta(t)$. As in the previous paragraph, there exists $\alpha<\lambda$ such that $\beta(t) \leq \alpha$ for all $t$. This shows that the representatives of $f$ and $g$ are in fact equal after passing to $\lim _{t} F_{\alpha}^{t}$, which shows that $f$ equals $g$.

Corollary 3.5 Every object of every pro-category is $\kappa$-cosmall relative to all pro-maps for some $\kappa$.

Proof This follows immediately from Proposition 3.3.

Corollary 3.6 Let $\mathcal{C}$ be any category, and let $\omega$ be the first infinite ordinal. Every constant ind-object of ind-C $\mathcal{C}$ is $\omega$-small relative to all ind-maps. More generally, if $X$ is an ind-object and $\kappa$ is an infinite regular cardinal such that $X$ can be represented by a diagram with fewer than $\kappa$ morphisms, then $X$ is $\kappa$-small relative to all ind-maps. Therefore, every object of every ind-category is $\kappa$-small relative to all ind-maps for some $\kappa$.

Proof Using that the category ind- $\mathcal{C}$ is isomorphic to the opposite of the category pro- $\left(\mathcal{C}^{\mathrm{op}}\right)$, the corollary is dual to Proposition 3.3 and Corollary 3.5 .

While these results seem innocuous, they have allowed us to provide a slick proof of Theorem 6.5. whose proof originally occupied many pages.

\subsection{Strict Model Structures}

Let $\mathcal{C}$ be a proper simplicial model category, such as the category of topological spaces, simplicial sets, or any of the standard models for spectra. Then the category pro- $\mathcal{C}$ has a strict model structure as originally developed in [10. See [19] for details about this. The role of properness is to replace the awkward niceness hypothesis of [10, §2.3]. The strict weak equivalences (resp., strict cofibrations) of pro- $\mathcal{C}$ are the essentially levelwise weak equivalences (resp., cofibrations). The strict fibrations of pro- $\mathcal{C}$ are defined by the right lifting property with respect to strict acyclic cofibrations. In fact, a more explicit description of the fibrations (up to retract) is possible [19, Defn. 4.2], but we do not need this.

Dually, ind-C $\mathcal{C}$ also has a strict structure. The strict weak equivalences (resp., strict fibrations) of ind-C $\mathcal{C}$ are the essentially levelwise weak equivalences (resp., 
fibrations), and the strict cofibrations of ind- $\mathcal{C}$ are defined by the left lifting property with respect to acyclic fibrations.

The simplicial structure is defined in the following way. For any two proobjects $X$ and $Y$, the simplicial mapping space $\operatorname{Map}_{\text {pro }}(X, Y)$ is defined to be $\lim _{s} \operatorname{colim}_{t} \operatorname{Map}\left(X_{t}, Y_{s}\right)$. Dually, if $X$ and $Y$ are ind-objects, then the simplicial mapping space $\operatorname{Map}_{\text {ind }}(X, Y)$ is defined to be $\lim _{t} \operatorname{colim}_{s} \operatorname{Map}\left(X_{t}, Y_{s}\right)$.

Tensors and cotensors with finite simplicial sets are defined levelwise. Tensors and cotensors with arbitrary simplicial sets are defined by extending with limits and colimits from the definition for finite simplicial sets. See [16, Defn. 16.2] for the reason for this complication with infinite simplicial sets.

Suppose that $\mathcal{C}$ is stable in the sense that $\mathcal{C}$ is a pointed model category in which the suspension and loops functors form a Quillen equivalence from $\mathcal{C}$ to itself. Then the strict model structures on pro- $\mathcal{C}$ and ind- $\mathcal{C}$ are again stable. Suspensions and loops in pro- $\mathcal{C}$ and ind $\mathcal{C}$ are defined levelwise.

We will need the following result about strict weak equivalences and essentially levelwise properties later.

Proposition 3.7 Let $\mathcal{C}$ be a proper simplicial model category, and let $C$ be any class of fibrant objects of $\mathcal{C}$ that is closed under weak equivalences between fibrant objects. If $f: X \rightarrow Y$ is a strict weak equivalence such that $Y$ is strictly fibrant and $X$ belongs to $C$ essentially levelwise, then $Y$ also belongs to $C$ essentially levelwise.

Proof We may assume that $f$ is a levelwise weak equivalence and that there is an isomorphism $X \rightarrow Z$ such that $Z$ belongs to $C$ levelwise. We may take a level replacement for the diagram $Z \leftarrow X \rightarrow Y$ in such a way that $Z$ still belongs to $C$ levelwise and $X \rightarrow Y$ is still a levelwise weak equivalence 4. A.3.2]. Moreover we may assume that the indexing category is a cofinite directed set (see [19, $\S 2.3]$ ). Factor the level map $X \rightarrow Z$ into a levelwise cofibration $X \rightarrow A$ followed by a levelwise acyclic fibration $A \rightarrow Z$. Note that this is not a factorization in the strict model structure; it is simply a factorization of each map $X_{s} \rightarrow Z_{s}$ in a functorial way.

Now let $P$ be the levelwise pushout $A \coprod_{X} Y$, and let $Q$ be the levelwise pushout $Z \coprod_{X} Y$. The map $A \rightarrow P$ is a cobase change of a levelwise weak equivalence along a levelwise cofibration, so it is also a levelwise weak equivalence.

The natural map $Y \rightarrow Q$ is an isomorphism in pro- $\mathcal{C}$ because it is a cobase change of an isomorphism. Also, this map factors through $P$. Therefore, $Y$ is a retract of $P$. 
Now take a strict acyclic cofibration $P \rightarrow P^{\prime}$ such that $P^{\prime}$ is strictly fibrant. Since $P$ is indexed by a cofinite directed set, we may use the construction given in [19. Lem. 4.7] so that $P \rightarrow P^{\prime}$ is a levelwise weak equivalence and $P^{\prime}$ is levelwise fibrant. Now we have a diagram

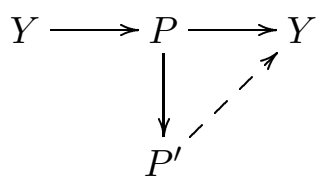

in which the dotted arrow exists because $P \rightarrow P^{\prime}$ is a strict acyclic cofibration and $Y$ is strictly fibrant. Therefore, $Y$ is also a retract of $P^{\prime}$. The class of pro-objects that belong to $C$ essentially levelwise is closed under retract 17, Thm. 5.5], so it suffices to consider $P^{\prime}$.

For each $s$, there is a zig-zag

$$
Z_{s} \leftarrow A_{s} \rightarrow P_{s} \rightarrow P_{s}^{\prime}
$$

of weak equivalences. Since $Z_{s}$ belongs to $C$, the assumption on $C$ implies that each $P_{s}^{\prime}$ belongs to $C$.

Remark 3.8 Let $\mathcal{C}$ be a proper simplicial model category, and let $C$ be any class of objects of $\mathcal{C}$ that is closed under weak equivalences. Then strict weak equivalences preserve the class of objects in pro- $\mathcal{C}$ that belong to $C$ essentially levelwise. The proof of this fact is basically the same as the proof of Proposition 3.7 but slightly shorter.

\section{Localizations of Pro-Categories}

In this section, we prove a general localization result for homotopy theories of pro-categories. The reason that we obtain localizations, not colocalizations, is that we apply the dual of Theorem 2.6 Let $\mathcal{C}$ be a proper simplicial model category. Let $K$ be any set of fibrant objects in $\mathcal{C}$, and let $c K$ be the set of constant pro-objects $c A$ such that $A$ belongs to $K$.

Definition 4.1 A map in pro- $\mathcal{C}$ is a cofibration if it is an essentially levelwise cofibration.

These cofibrations are exactly the strict cofibrations of pro- $\mathcal{C}$. 
Definition 4.2 A map $f: X \rightarrow Y$ in pro- $\mathcal{C}$ is a $c K$-local weak equivalence if it induces a weak equivalence

$$
\operatorname{Map}_{\text {pro }}(\tilde{X}, c A)=\operatorname{colim}_{s} \operatorname{Map}_{\mathcal{C}}\left(\tilde{X}_{s}, A\right) \rightarrow \operatorname{colim}_{t} \operatorname{Map}_{\mathcal{C}}\left(\tilde{Y}_{t}, A\right)=\operatorname{Map}_{\text {pro }}(\tilde{Y}, c A)
$$

for all $A$ in $K$, where $\tilde{X}$ and $\tilde{Y}$ are strictly cofibrant replacements for $X$ and $Y$.

Definition 4.3 A map in pro- $\mathcal{C}$ is a $c K$-local fibration if it has the right lifting property with respect to all $c K$-local acyclic cofibrations.

Theorem 4.4 Let $\mathcal{C}$ be a proper simplicial model category, and let $K$ be any set of fibrant objects in $\mathcal{C}$. Definitions 4.1 4.2 and 4.3 define a left proper simplicial model structure on pro-C .

Proof This is an application of a dual version of Theorem 2.6] in which the objects of $K$ are required to be cosmall and cofibrations are preserved by sequential limits. These hypotheses are proved in Proposition 3.3 and 17, Cor. 5.4].

We emphasize that it is not necessary that the model category $\mathcal{C}$ satisfies Hypothesis 2.4 in Theorem 4.4. It is important that pro- $\mathcal{C}$ does satisfy this hypothesis. This happens by general arguments about pro-categories, not by using specific properties of $\mathcal{C}$. Theorem 4.4 could be stated even more generally. There is no need to localize with respect only to constant pro-objects, since every pro-object is cosmall for some cardinal (see Corollary [3.5). Theorem 6.5 below is one example of the situation in Theorem 4.4. See 20] for other examples.

We really do need the dual of Theorem 2.6 in order to establish the $c K$-local model structure of Theorem [4.4, the dual of [12, Thm. 5.1.1] is not strong enough. The problem is that the strict model structure for pro- $\mathcal{C}$ is not fibrantly generated in general. See [19, §5] for a proof that the strict model structure for pro-simplicial sets is not fibrantly generated.

Remark 4.5 Suppose that $\mathcal{C}$ is a stable model category in the sense that the loops and suspension functors are inverse Quillen equivalences of $\mathcal{C}$ with itself. Let $K$ be a set of fibrant objects of $\mathcal{C}$ such that for every $A$ in $K, \Omega A$ and $\Sigma \tilde{A}$ are weakly equivalent to elements of $K$, where $\tilde{A}$ is a cofibrant replacement for $A$. In other words, $K$ is closed, up to homotopy, under suspensions and loops. Then it can be proved that the $c K$-local model structure is also stable. We will not need this result, but the model structure of Theorem 6.5 is an example of this situation. 
We do not know whether the $c K$-local model structure on pro- $\mathcal{C}$ is always right proper, even though we are always assuming that $\mathcal{C}$ is proper. If we assume in addition that $\mathcal{C}$ is stable, then we can show that the model structure is right proper.

Proposition 4.6 If $\mathcal{C}$ is a stable proper simplicial model category and $K$ is a set of fibrant objects of $\mathcal{C}$, then the $c K$-local model structure is right proper.

Proof Suppose given a pullback square

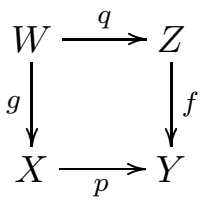

in pro- $\mathcal{C}$ in which $p$ is a $c K$-local fibration and $f$ is a $c K$-local weak equivalence. We want to show that $g$ is also a $c K$-local weak equivalence.

Let $F$ be the homotopy fibre of $p$ with respect to the strict model structure, which is also the homotopy fibre of $q$. Now $\Sigma \tilde{F}$ is the homotopy cofibre of both $p$ and $q$, where $\tilde{F}$ is a cofibrant replacement for $F$. We have a diagram

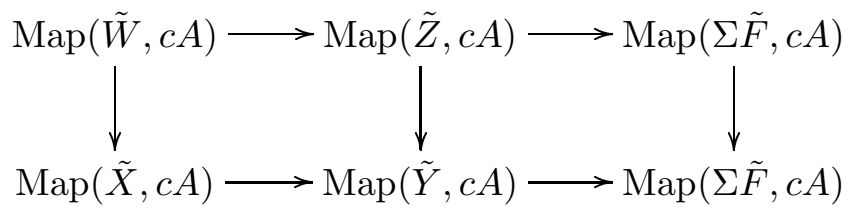

of simplicial sets in which the rows are fibre sequences. Here $\tilde{W}, \tilde{Z}, \tilde{X}$, and $\tilde{Y}$ are cofibrant replacements for $W, Z, X$, and $Y$, and $A$ is any object of $K$. The second and third vertical maps are weak equivalences, which means that the first vertical map is also.

\subsection{Fibrant Pro-Objects}

Later we shall need a more explicit description of $c K$-local fibrant pro-objects. This section contains this description. We are still assuming that $\mathcal{C}$ is a proper simplicial model category and that each $A$ in $K$ is fibrant.

Definition 4.7 The class of $K$-nilpotent objects of $\mathcal{C}$ is the smallest class of fibrant objects such that:

(1) the terminal object of $\mathcal{C}$ is $K$-nilpotent; 
(2) weak equivalences between fibrant objects preserve $K$-nilpotence;

(3) and if $X$ is $K$-nilpotent, $A$ belongs to $K$, and $X \rightarrow A^{\partial \Delta[n]}$ is any map, then the fibre product $X \times_{A^{\partial \Delta[n]}} A^{\Delta[n]}$ is again $K$-nilpotent.

In other words, an object is $K$-nilpotent if and only if it can be built, up to weak equivalence, from the terminal object by a finite sequence of base changes of maps of the form $A^{\Delta[n]} \rightarrow A^{\partial \Delta[n]}$ with $A$ in $K$. The terminology arises from the connection with nilpotent spaces when $\mathcal{C}$ is the category of simplicial sets and $K$ is the collection of Eilenberg-Mac Lane spaces. See [20] for details.

In this paper, the only important example occurs with $\mathcal{C}$ the category of spectra and $K$ the set of spheres. In this specific case, we give in Proposition [6.6 a more concrete description of $K$-nilpotent spectra.

Lemma 4.8 Let $f: X \rightarrow Y$ be any $c K$-local weak equivalence between cofibrant pro-objects. Then the map $\operatorname{Map}(f, c Z): \operatorname{Map}(Y, c Z) \rightarrow \operatorname{Map}(X, c Z)$ is a weak equivalence for all $K$-nilpotent objects $Z$ of $\mathcal{C}$.

Proof The map $\operatorname{Map}(f, c Z)$ is a weak equivalence (even an isomorphism) when $Z=*$. Since $X$ and $Y$ are cofibrant and $c Z$ is strictly fibrant, the weak homotopy types of $\operatorname{Map}(Y, c Z)$ and $\operatorname{Map}(X, c Z)$ do not depend on the choice of $Z$ up to weak equivalence.

It only remains to consider condition (3) of Definition 4.7. Suppose for induction that $Z$ is $K$-nilpotent and that $\operatorname{Map}(Y, c Z) \rightarrow \operatorname{Map}(X, c Z)$ is a weak equivalence. Let $Z^{\prime}$ be the fibre product $Z \times_{A^{\partial \Delta[k]}} A^{\Delta[k]}$ for some object $A$ in $K$. Since $A^{\Delta[k]} \rightarrow A^{\partial \Delta[k]}$ is a fibration, this fibre product is actually a homotopy fibre product, which means that $\operatorname{Map}\left(Y, c Z^{\prime}\right)$ is the homotopy fibre product of the top row in the diagram

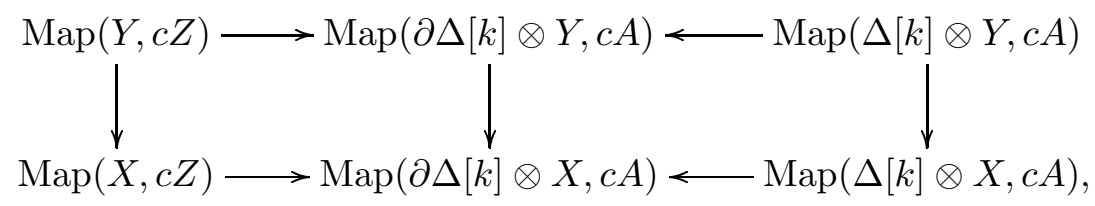

and $\operatorname{Map}\left(X, c Z^{\prime}\right)$ is the homotopy fibre product of the bottom row. The left vertical map is a weak equivalence by the induction assumption, and the other two vertical maps are weak equivalences because the $c K$-local model structure is simplicial and because $f$ is a $c K$-local weak equivalence. Thus, the induced map on homotopy fibre products is also a weak equivalence. 
Proposition 4.9 An object $X$ of pro-C is $c K$-local fibrant if and only if it is strictly fibrant and essentially levelwise $K$-nilpotent.

That $X$ is essentially levelwise $K$-nilpotent means that $X$ is isomorphic to a pro-object $Y$ such that each $Y_{s}$ is $K$-nilpotent.

Proof First suppose that $X$ is $c K$-local fibrant. Every $c K$-local fibration is a strict fibration, so $X$ is strictly fibrant. It remains to show that $X$ is essentially levelwise $K$-nilpotent. By applying a functorial cofibrant replacement construction levelwise to $X$, we get a map $\tilde{X} \rightarrow X$ which is a levelwise weak equivalence such that $\tilde{X}$ is levelwise cofibrant (and, in particular, strictly cofibrant). If we can show that $\tilde{X}$ is essentially levelwise $K$-nilpotent, then we can use Proposition 3.7 to conclude that $X$ is also essentially levelwise $K$-nilpotent. In other words, we might as well assume that $X$ is strictly cofibrant.

Consider the factorization $X \rightarrow W \rightarrow c *$ of the map $X \rightarrow c *$ as described in the dual to the proof of Lemma 2.5. so $W$ is $c K$-local fibrant. Note that each $c A$ is $\omega$-cosmall by Proposition 3.3 Therefore, we may take $\kappa$ to be $\omega$, and there are no limit ordinals in the construction of $W$.

Since $X$ is strictly cofibrant, the dual of Lemma 2.3. (c) tells us that the map $X \rightarrow W$ is a $c K$-local acyclic cofibration. Hence $X$ is a retract of $W$ because $X$ is $c K$-local fibrant. The class of pro-objects having any property essentially levelwise is closed under retracts [17, Thm. 5.5], so it suffices to consider $W$. But the class of pro-objects having any property essentially levelwise is also closed under cofiltered limits [17, Thm. 5.1], so it suffices to consider each $W_{\beta}$. Assume for induction that the pro-object $W_{\beta-1}$ is levelwise $K$-nilpotent. We may take a level representation for the diagram

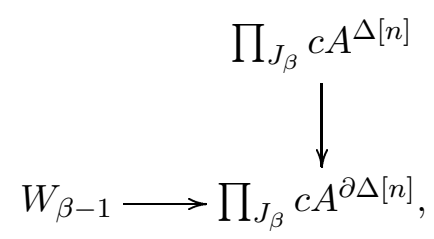

and we construct $Z_{\beta}$ by taking the levelwise fibre product. In fact, it is possible to construct the level representation in such a way that the replacement for $W_{\beta-1}$ is a diagram of objects that already appeared in the original $W_{\beta-1}$. This means that the new $W_{\beta-1}$ is still levelwise $K$-nilpotent.

The construction of arbitrary products in pro-categories [16, Prop. 11.1] shows that the map

$$
\prod_{J_{\beta}} c A^{\Delta[n]} \rightarrow \prod_{J_{\beta}} c A^{\partial \Delta[n]}
$$


is levelwise a finite product of maps of the form

$$
A^{\Delta[n]} \rightarrow A^{\partial \Delta[n] .}
$$

It follows immediately that $Z_{\beta}$ is levelwise $K$-nilpotent. Now $W_{\beta} \rightarrow Z_{\beta}$ is a levelwise weak equivalence, so $W_{\beta}$ is also levelwise $K$-nilpotent. This finishes one implication.

Now suppose that $X$ is essentially levelwise $K$-nilpotent and strictly fibrant. We may assume that each $X_{s}$ is $K$-nilpotent. Using the lifting property characterization of $c K$-local fibrant pro-objects, we must show that the map

$$
f: \operatorname{Map}(B, X) \rightarrow \operatorname{Map}(A, X)
$$

is an acyclic fibration of simplicial sets for every $c K$-local acyclic cofibration $i: A \rightarrow B$ in pro- $\mathcal{C}$. In fact, by [12, Prop. 13.2.1], we may further assume that $A$ and $B$ are cofibrant pro-objects (using that the $c K$-local model structure is left proper). We already know that $f$ is a fibration because of the strict model structure. Since $X$ is strictly fibrant, $f$ is weakly equivalent to $\operatorname{holim}_{s} f_{s}$, where $f_{s}$ is the map

$$
f_{s}: \operatorname{Map}\left(B, c X_{s}\right) \rightarrow \operatorname{Map}\left(A, c X_{s}\right) .
$$

Therefore, we need only show that each $f_{s}$ is a weak equivalence. This is true by Lemma 4.8

Remark 4.10 In Definition 4.7 one might also require that the class of $K$ nilpotent objects is closed under retracts. Strangely, this makes no difference in Proposition 4.9. The statement of that proposition is true exactly as worded, whether or not $K$-nilpotent objects are closed under retracts. This surprising phenomenon arises from the surprising way in which retracts interact with essentially levelwise properties of pro-objects [17, Thm. 5.5].

\subsection{Ind-Categories}

All the results of this section dualize to ind-categories. More specifically, let $K$ be a set of cofibrant objects in a proper simplicial model category $\mathcal{C}$. A map $f: X \rightarrow Y$ in ind- $\mathcal{C}$ is a $c K$-colocal weak equivalence if it induces a weak equivalence

$$
\operatorname{Map}_{\text {ind }}(c A, \hat{X})=\operatorname{colim}_{s} \operatorname{Map}_{\mathcal{C}}\left(A, \hat{X}_{s}\right) \rightarrow \operatorname{colim}_{t} \operatorname{Map}\left(A, \hat{Y}_{t}\right)=\operatorname{Map}_{i n d}(c A, \hat{Y})
$$

for all $A$ in $K$, where $\hat{X}$ and $\hat{Y}$ are strictly fibrant replacements for $X$ and $Y$. Fibrations are just strict fibrations, and $c K$-colocal cofibrations are defined 
by a lifting property. These definitions give a right proper $c K$-colocal model structure on ind- $\mathcal{C}$. If $\mathcal{C}$ is stable, then this model structure is also left proper. An ind-object is $c K$-colocal cofibrant if and only if it is strictly cofibrant and, up to isomorphism, it is levelwise weakly equivalent to an object that can be built out of the initial object by a finite sequence of cofibrant homotopy pushouts of maps of the form $\partial \Delta[n] \otimes A \rightarrow \Delta[n] \otimes A$.

\section{$5 \quad$ Preliminaries on Spectra}

Now we review some definitions and results about spectra. We work in the category of symmetric spectra 14. This category has a proper simplicial cofibrantly generated stable model structure. We take this structure as the "standard" model structure for spectra. Whenever we write "spectrum", we always mean "symmetric spectrum". We write $S^{n}$ for a fixed cofibrant and fibrant model for the $n$th suspension of the sphere spectrum.

The category of symmetric spectra is closed symmetric monoidal. This means that it has an associative commutative unital smash product $\wedge$ and an internal function object $F(\cdot, \cdot)$ such that $F(Z, \cdot)$ is right adjoint to $\cdot \wedge Z$. That is, there is a bijection between maps $X \rightarrow F(Z, Y)$ and maps $X \wedge Z \rightarrow Y$.

We shall use the following model theoretic property of the functor $F(\cdot, Y)$ when $Y$ is an arbitrary fixed fibrant spectrum [14, Cor. 5.3.9]. Namely, $F(\cdot, Y)$ takes cofibrations to fibrations. More precisely, if $i: A \rightarrow B$ is a cofibration, then

$$
F(i, Y): F(B, Y) \rightarrow F(A, Y)
$$

is a fibration.

The weak equivalences in the category of spectra are defined in [14, Defn. 3.1.3]. The stable homotopy category is the category obtained by inverting these maps, which are also called stable equivalences. We will not repeat the definition of weak equivalence here, but using the following definition of homotopy groups we will state an equivalent condition below.

Definition 5.1 For any spectrum $X$, let $\pi_{n} X$ be the set $\left[S^{n}, X\right]$ of maps in the stable homotopy category.

When $X$ is a fibrant spectrum, $\pi_{n} X$ is isomorphic to the traditional $n$th stable homotopy group $\operatorname{colim}_{k} \pi_{n+k} X_{k}$. More generally, we can calculate $\pi_{n} X$ by considering the traditional $n$th stable homotopy group of a fibrant replacement 
for $X$. Weak equivalences are not defined in terms of homotopy groups because the definition of homotopy groups depends on the prior existence of the stable homotopy category. Nevertheless, the homotopy groups do detect stable weak equivalences of symmetric spectra in the sense that a map $f: X \rightarrow Y$ is a stable equivalence if and only if $\pi_{n} f$ is an isomorphism for every $n \in \mathbb{Z}$.

Filtered colimits preserve fibrant spectra since the generating acyclic cofibrations have compact domains [14, Defn. 3.4.9] (recall that an object $C$ of a category is compact if $\operatorname{Hom}(C, \cdot)$ commutes with filtered colimits). Since filtered colimits also preserve the traditional stable homotopy groups, it follows that

$$
\pi_{n}\left(\operatorname{colim}_{s} X_{s}\right) \cong \operatorname{colim}_{s}\left(\pi_{n} X_{s}\right)
$$

for every filtered diagram $X$ of spectra.

\section{$6 \quad \pi^{*}$-Model Structure on Pro-Spectra}

In this section we specialize Theorem 4.4 to describe a model structure on the category of pro-spectra that involves cohomotopy. Later we compare the associated homotopy theory to ordinary stable homotopy theory.

Definition 6.1 A map of pro-spectra is a cofibration if it is an essentially levelwise cofibration.

These cofibrations are identical with strict cofibrations of pro-spectra.

Recall that the cohomotopy group $\pi^{n} X$ of a spectrum $X$ is the group of stable weak homotopy classes $\left[X, S^{n}\right]$. Thus $\pi^{*}$ is the cohomology theory represented by the sphere spectrum $S^{0}$.

Definition 6.2 A map $f: X \rightarrow Y$ of pro-spectra is a $\pi^{*}$-weak equivalence if it induces an isomorphism $\operatorname{colim}_{s} \pi^{n} Y_{s} \rightarrow \operatorname{colim}_{t} \pi^{n} X_{t}$ for every $n \in \mathbb{Z}$.

It is important that we are not requiring that $\pi^{n} X \rightarrow \pi^{n} Y$ be an ind-isomorphism. Non-isomorphic ind-abelian groups may have isomorphic colimits when one allows infinitely generated abelian groups. It is also important that we are not using the groups $\pi^{n} \lim _{s} X_{s}$ or $\pi^{n} \operatorname{holim}_{s} X_{s}$. In general, very little can be said about these groups in terms of the groups $\pi^{n} X_{s}$. 
Proposition 6.3 Let $f: X \rightarrow Y$ be a map of pro-spectra, and let $\tilde{f}: \tilde{X} \rightarrow \tilde{Y}$ be a strictly cofibrant replacement for $f$. The following conditions are equivalent:

(1) $f$ is a $\pi^{*}$-weak equivalence;

(2) $\operatorname{Map}\left(\tilde{f}, c S^{n}\right): \operatorname{Map}\left(\tilde{Y}, c S^{n}\right) \rightarrow \operatorname{Map}\left(\tilde{X}, c S^{n}\right)$ is a weak equivalence of simplicial sets for every $n \in \mathbb{Z}$.

(3) $\operatorname{colim}_{t} F\left(\tilde{Y}_{t}, S^{0}\right) \rightarrow \operatorname{colim}_{s} F\left(\tilde{X}_{s}, S^{0}\right)$ is a weak equivalence of spectra.

Proof For any cofibrant pro-spectrum $Z$ and any $k \geq 0$,

$$
\pi_{k} \operatorname{Map}\left(Z, c S^{n}\right)=\pi_{k} \operatorname{colim}_{t} \operatorname{Map}\left(Z_{t}, S^{n}\right)=\underset{t}{\operatorname{colim}}\left[Z_{t}, S^{n-k}\right] .
$$

The case $k=0$ tells us that condition (2) implies condition (1).

Now suppose that $f$ is a $\pi^{*}$-weak equivalence. From the computation of the previous paragraph, we know that $\operatorname{Map}\left(\tilde{f}, c S^{n}\right)$ induces an isomorphism on all homotopy groups at the canonical basepoints. Since $\operatorname{Map}\left(\tilde{f}, c S^{n}\right)$ is weakly equivalent to the loop space $\Omega \operatorname{Map}\left(\tilde{f}, c S^{n+1}\right)$, we only need to compute homotopy groups at one basepoint. This shows that condition (1) implies condition (2).

For condition (3), note that $\pi_{k} \operatorname{colim}_{t} F\left(\tilde{X}, S^{0}\right)$ is isomorphic to $\operatorname{colim}_{t} \pi^{-k} X_{t}$ (and similarly for $\tilde{Y}$ ). This shows that condition (3) is equivalent to condition (1).

Definition 6.4 A map of pro-spectra is a $\pi^{*}$-fibration if it has the right lifting property with respect to all $\pi^{*}$-acyclic cofibrations.

Theorem 6.5 Definitions 6.1, 6.2, and 6.4 define a proper simplicial model structure on the category of pro-spectra.

We call this the $\pi^{*}$-model structure on pro-spectra.

Proof Proposition 6.3 tells us that we are discussing a $c K$-localization, where $K$ is the set of spheres. Therefore, Theorem 4.4 gives us everything but right properness. Since the model category of spectra is stable (see the last paragraph of Section 3), Proposition 4.6 gives us right properness.

Now we will identify the $\pi^{*}$-fibrant pro-spectra. We say that a spectrum is homotopy-finite if it is weakly equivalent to a finite complex, i.e. if its image in the stable homotopy category is in the thick subcategory generated by $S^{0}$. 
Proposition 6.6 Let $K$ be the set of spheres. A spectrum is $K$-nilpotent (see Definition 4.7) if and only if it is fibrant and homotopy-finite.

Proof First suppose that $X$ is $K$-nilpotent. We work by induction over the number of pullbacks in the construction of $X$, noting that the terminal object is homotopy-finite and weak equivalences preserve homotopy-finiteness.

To do the inductive step, assume that $X$ equals $Y \times{ }_{\left(S^{k}\right)^{\partial \Delta[n]}}\left(S^{k}\right)^{\Delta[n]}$, where $Y$ is homotopy-finite. We have to show that $X$ is also homotopy-finite. Each of the spectra $Y,\left(S^{k}\right)^{\partial \Delta[n]}$, and $\left(S^{k}\right)^{\Delta[n]}$ is homotopy-finite, and the map $\left(S^{k}\right)^{\Delta[n]} \rightarrow\left(S^{k}\right)^{\partial \Delta[n]}$ is a fibration. Therefore, $X$ is a homotopy fibre product of three homotopy-finite spectra, so $X$ is also homotopy-finite.

Now assume that $X$ is fibrant and homotopy-finite. We have to show that $X$ is $K$-nilpotent. We induct on the number of cells in $X$. If $X$ is weakly contractible, then it is $K$-nilpotent by definition. To do the inductive step, suppose that there is a fibre sequence

$$
X \rightarrow Y \rightarrow S^{k},
$$

where $X$ has one more cell than $Y$ and $Y$ is $K$-nilpotent. (This is dual to the usual way of attaching cells, but produces the same class of finite complexes because of Spanier-Whitehead duality.)

We claim that there is a homotopy pullback square

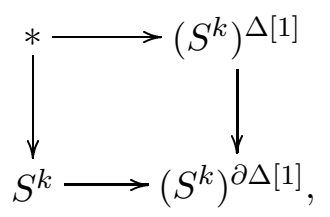

where the bottom horizontal map is inclusion into the first factor. See the following lemma for the proof. In the diagram

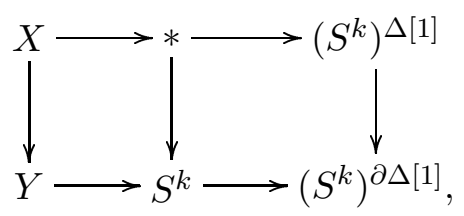

the left square is also a homotopy pullback square since $X$ is the homotopy fibre of $Y \rightarrow S^{k}$. Thus the composite square is also a homotopy pullback square, which means that $X$ is $K$-nilpotent. 
Lemma 6.7 Let $f: S^{k} \rightarrow\left(S^{k}\right)^{\partial \Delta[1]}=S^{k} \times S^{k}$ be the inclusion into the first factor. Then there is a homotopy pullback square

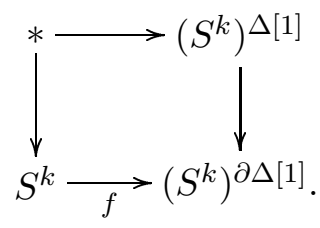

Proof Consider the pushout diagram

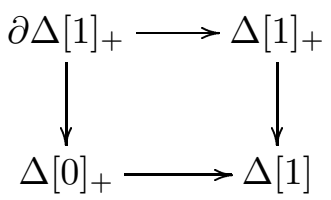

of pointed simplicial sets, where the top horizontal arrow is the obvious inclusion and the left vertical arrow takes 0 to 0 and takes both 1 and the basepoint to the basepoint. Note that $\Delta[1]$ is pointed at 1 .

If we apply the functor $\operatorname{Map}\left(\Sigma^{\infty}(-), S^{k}\right)$ to this diagram, we obtain a pullback square

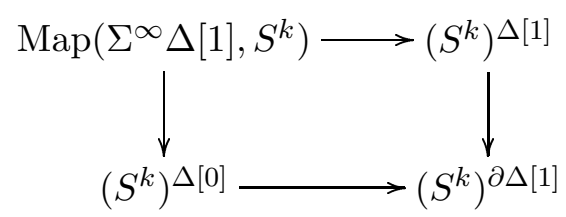

of spectra in which the upper right corner is contractible. This diagram is a homotopy pullback diagram because the right vertical map is a fibration.

Proposition 6.8 A pro-spectrum $X$ is $\pi^{*}$-fibrant if and only if it is essentially levelwise homotopy-finite and strictly fibrant.

This means that each $X_{s}$ has the weak homotopy type of a finite complex. There is no compatibility requirement for the weak equivalences between the spectra $X_{s}$ and the finite complexes.

Proof This follows immediately from Proposition 4.9 together with Proposition 6.6.

Remark 6.9 Using the above description of $\pi^{*}$-fibrant objects, it is possible to describe explicitly the total derived functor Rlim of the limit functor from 
pro-spectra to spectra. This functor is computed by taking the limit of a $\pi^{*}$ fibrant replacement. With the strict structure on pro-spectra, Rlim is just the homotopy limit functor. However, with the $\pi^{*}$-model structure, Rlim is related to Spanier-Whitehead duality.

Let $X$ be a spectrum; we shall calculate $\operatorname{Rlim}(c X)$. Take a $\pi^{*}$-fibrant replacement $\hat{X}$ for $c X$. From Proposition 4.9 we know that each spectrum in $\hat{X}$ is homotopy-finite; thus, $\hat{X}$ is levelwise weakly equivalent to $F\left(F\left(\hat{X}, S^{0}\right), S^{0}\right)$. Therefore, $\operatorname{Rlim}(c X)$ is weakly equivalent to $\operatorname{holim}_{s} F\left(F\left(\hat{X}_{s}, S^{0}\right), S^{0}\right)$, which is equivalent to $F\left(\operatorname{colim}_{s} F\left(\hat{X}_{s}, S^{0}\right), S^{0}\right)$. A computation of homotopy groups shows that $\pi_{n} \operatorname{colim}_{s} F\left(\hat{X}_{s}, S^{0}\right)$ equals $\pi^{-n} X$, so $\operatorname{colim}_{s} F\left(\hat{X}_{s}, S^{0}\right)$ is weakly equivalent to the ordinary Spanier-Whitehead dual $F\left(X, S^{0}\right)$ of $X$. Thus, we have shown that $\operatorname{Rlim}(c X)$ is equivalent to $F\left(F\left(X, S^{0}\right), S^{0}\right)$.

A similar analysis shows that if $X$ is an arbitrary pro-spectrum, then $\operatorname{Rlim} X$ is equivalent to $F\left(\operatorname{colim}_{s} F\left(X_{s}, S^{0}\right), S^{0}\right)$, i.e., the Spanier-Whitehead dual of the colimit of the levelwise Spanier-Whitehead dual of $X$.

\section{$7 \quad \pi_{*}$-Model Structure on Ind-Spectra}

Now we proceed to ind-spectra. All of the following definitions and results are dual to analogous results in the previous section. We skip the proofs because they are no different.

Definition 7.1 A map of ind-spectra is a fibration if it is an essentially levelwise fibration.

These fibrations are identical with strict fibrations of ind-spectra.

Definition 7.2 A map of ind-spectra $X \rightarrow Y$ is a $\pi_{*}$-weak equivalence if for every $n \in \mathbb{Z}$, the map $\operatorname{colim}_{s} \pi_{n} X_{s} \rightarrow \operatorname{colim}_{t} \pi_{n} Y_{t}$ is an isomorphism.

Proposition 7.3 Let $f: X \rightarrow Y$ be a map of ind-spectra, and let $\hat{f}: \hat{X} \rightarrow \hat{Y}$ be a strictly fibrant replacement for $f$. The following conditions are equivalent:

(1) $f$ is a $\pi_{*}$-weak equivalence;

(2) $\operatorname{Map}\left(c S^{n}, \hat{f}\right): \operatorname{Map}\left(c S^{n}, \hat{Y}\right) \rightarrow \operatorname{Map}\left(c S^{n}, \hat{X}\right)$ is a weak equivalence of simplicial sets for every $n \in \mathbb{Z}$;

(3) $\operatorname{colim}_{s} X_{s} \rightarrow \operatorname{colim}_{t} Y_{t}$ is a stable weak equivalence of spectra. 
Definition 7.4 A map of ind-spectra is a $\pi_{*}$-cofibration if it has the left lifting property with respect to all $\pi_{*}$-acyclic fibrations.

Theorem 7.5 Definitions 7.1, 7.2, and 7.4 define a proper simplicial model structure on the category of ind-spectra.

We call this the $\pi_{*}$-model structure on ind-spectra.

Proof Everything but left properness is an application of the dual version of Theorem 4.4. Left properness follows in a manner dual to the proof of Proposition 4.6

Proposition 7.6 Consider the smallest class of cofibrant spectra such that:

(1) * belongs to the class;

(2) the class is closed under weak equivalences between cofibrant spectra;

(3) and if $X$ belongs to the class and $\partial \Delta[n] \otimes S^{k} \rightarrow X$ is any map, then the pushout $\Delta[n] \otimes S^{k} \coprod_{\partial \Delta[n] \otimes S^{k}} X$ also belongs to the class.

This class coincides with the class of cofibrant homotopy-finite spectra.

Proposition 7.7 An ind-spectrum $X$ is $\pi_{*}$-cofibrant if and only if it is essentially levelwise homotopy-finite and strictly cofibrant.

\section{Comparison of Homotopy Theories}

This section contains the main results of this paper. Namely, the homotopy category of pro-spectra is the opposite of the ordinary stable homotopy category. First, we study the homotopy theory of ind-spectra.

Theorem 8.1 The constant functor $c$ from spectra to ind-spectra is right adjoint to the functor colim. These functors form a Quillen equivalence when considering the $\pi_{*}$-model structure on ind-spectra.

Proof Let $X$ be an ind-spectrum, and let $Y$ be a spectrum. By direct calculation, $\operatorname{Hom}_{\text {ind }}(X, c Y) \cong \operatorname{Hom}(\operatorname{colim} X, Y)$. Thus $c$ and colim are adjoint.

The functor $c$ preserves fibrations and weak equivalences. Therefore, $c$ and colim are a Quillen pair.

By Proposition [7.3, $X \rightarrow c Y$ is a $\pi_{*}$-weak equivalence if and only if $\operatorname{colim}_{s} X_{s} \rightarrow$ $Y$ is a weak equivalence of spectra. Hence, $c$ and colim form a Quillen equivalence. 
According to Theorem 8.1 the homotopy category of ind-spectra is equivalent to the ordinary stable homotopy category. We suspect that this model structure is not cofibrantly generated, but we have not been able to prove it.

By Proposition 17.7, every ind-spectrum $X$ is $\pi_{*}$-weakly equivalent to an indspectrum whose objects are finite cell complexes. Therefore, the ordinary stable homotopy category is equivalent to a homotopy category of ind-(finite cell complexes), but this latter homotopy category does not arise from a model structure.

The next step is to compare the categories of pro-spectra and ind-spectra.

Lemma 8.2 The contravariant functor $F(\cdot, Y)$ from spectra to spectra is its own adjoint.

Proof A map $X \rightarrow F(Z, Y)$ corresponds to a map $X \wedge Z \rightarrow Y$. This corresponds to a map $Z \rightarrow F(X, Y)$ because $X \wedge Z$ and $Z \wedge X$ are isomorphic.

Let $Y$ be a fixed spectrum. By acting levelwise, the functor $F(\cdot, Y)$ induces a contravariant functor from pro-spectra to ind-spectra. It also induces a contravariant functor from ind-spectra to pro-spectra.

Proposition 8.3 Let $Y$ be an arbitrary fixed spectrum. The contravariant functors $F(\cdot, Y)$ from pro-spectra to ind-spectra and from ind-spectra to prospectra are adjoint in the sense that

$$
\operatorname{Hom}_{\text {pro }}(X, F(Z, Y)) \cong \operatorname{Hom}_{\text {ind }}(Z, F(X, Y))
$$

for every pro-spectrum $X$ and every ind-spectrum $Z$.

Proof This follows from direct computation and Lemma 8.2 .

Proposition 8.4 Let $Y$ be a homotopy-finite fibrant spectrum. Then the contravariant functors $F(\cdot, Y)$ from pro-spectra to ind-spectra and from indspectra to pro-spectra are a Quillen pair between the $\pi^{*}$-model structure on pro-spectra and the opposite of the $\pi_{*}$-model structure on ind-spectra.

Proof We already know that the functors are an adjoint pair by Proposition 8.3. In order to show that they are a Quillen pair, we must prove that $F(\cdot, Y)$ takes cofibrations (resp., $\pi^{*}$-acyclic cofibrations) of pro-spectra to fibrations (resp., $\pi_{*}$-acyclic fibrations) of ind-spectra. 
Since $Y$ is fibrant, $F(\cdot, Y)$ takes cofibrations of spectra to fibrations of spectra. Therefore, $F(\cdot, Y)$ takes levelwise cofibrations of pro-spectra to levelwise fibrations of ind-spectra. It follows that $F(\cdot, Y)$ takes essentially levelwise cofibrations of pro-spectra to essentially levelwise fibrations of ind-spectra.

Now let $i: A \rightarrow B$ be a $\pi^{*}$-acyclic cofibration of pro-spectra. Fix $k \in \mathbb{Z}$ and let $Z$ be a fibrant model for the spectrum $\Omega^{k} Y$. Since $Z$ is again homotopy-finite, the constant pro-spectrum $c Z$ is $\pi^{*}$-fibrant. Therefore, the map $\operatorname{Map}(B, c Z) \rightarrow$ $\operatorname{Map}(A, c Z)$ is an acyclic fibration of simplicial sets. In particular, the map $\pi_{0} \operatorname{colim}_{s} \operatorname{Map}\left(B_{s}, Z\right) \rightarrow \pi_{0} \operatorname{colim}_{t} \operatorname{Map}\left(A_{t}, Z\right)$ is an isomorphism. This means that $\pi_{0} \operatorname{colim}_{s} F\left(B_{s}, Z\right) \rightarrow \pi_{0} \operatorname{colim}_{t} F\left(A_{t}, Z\right)$ is also an isomorphism (because $\pi_{0} F(C, D)=\pi_{0} \operatorname{Map}(C, D)$ for any spectra $C$ and $D$ and because $\pi_{0}$ commutes with filtered colimits).

Since $\pi_{0} \operatorname{colim}_{s} F\left(B_{s}, Z\right)$ is isomorphic to $\pi_{k} \operatorname{colim}_{s} F\left(B_{s}, Y\right)$ (and similarly for $A$ ), it follows that $\operatorname{colim}_{s} F\left(B_{s}, Y\right) \rightarrow \operatorname{colim}_{t} F\left(A_{t}, Y\right)$ is a weak equivalence of spectra. Proposition 7.3 tells us that the map $F(i, Y): F(B, Y) \rightarrow F(A, Y)$ is a $\pi_{*}$-weak equivalence of ind-spectra.

Theorem 8.5 The contravariant functors $F\left(\cdot, S^{0}\right)$ from pro-spectra to indspectra and from ind-spectra to pro-spectra form a Quillen equivalence between the $\pi^{*}$-model structure on pro-spectra and the opposite of the $\pi_{*}$-model structure on ind-spectra.

Proof We already showed in Proposition 8.4 that the functors are a Quillen pair. Note that the hypothesis of Proposition 8.4 is satisfied because $S^{0}$ is homotopy-finite.

Let $X$ be a cofibrant pro-spectrum, let $Z$ be a cofibrant ind-spectrum, and let $f: X \rightarrow F\left(Z, S^{0}\right)$ be a map of pro-spectra. Our goal is to show that $f$ is a $\pi^{*}$-weak equivalence if and only if the adjoint map $Z \rightarrow F\left(X, S^{0}\right)$ is a $\pi_{*}$-weak equivalence of ind-spectra.

By Proposition 6.3 the map $f$ is a $\pi^{*}$-weak equivalence if and only if the map

$$
\underset{s}{\operatorname{colim}} F\left(\tilde{F}\left(Z_{s}, S^{0}\right), S^{0}\right) \rightarrow \operatorname{colim}_{t} F\left(X, S^{0}\right)
$$

is a weak equivalence of spectra. Here $\tilde{F}(C, D)$ refers to a cofibrant replacement for the function spectrum $F(C, D)$.

By Proposition [7.7 we may assume that each $Z_{s}$ is homotopy-finite. Since the Spanier-Whitehead double dual of a finite complex is itself, the map $Z \rightarrow$ $F\left(\tilde{F}\left(Z, S^{0}\right), S^{0}\right)$ is a levelwise weak equivalence. In particular, the map

$$
\underset{s}{\operatorname{colim}} Z_{s} \rightarrow \underset{s}{\operatorname{colim}} F\left(\tilde{F}\left(Z_{s}, S^{0}\right), S^{0}\right)
$$


is a weak equivalence of spectra.

The previous two paragraphs imply that $f$ is a weak equivalence if and only if the composition

$$
\underset{s}{\operatorname{colim}} Z_{s} \rightarrow \operatorname{colim}_{s} F\left(\tilde{F}\left(Z_{s}, S^{0}\right), S^{0}\right) \rightarrow \operatorname{colim}_{t} F\left(X, S^{0}\right)
$$

is a weak equivalence of spectra. By Proposition 7.3, this last map is a weak equivalence if and only if the map $Z \rightarrow F\left(X, S^{0}\right)$ is a $\pi_{*}$-weak equivalence of ind-spectra.

Corollary 8.6 (Main result) The category of pro-spectra with its $\pi^{*}$-model structure is Quillen equivalent to the opposite of the category of symmetric spectra with its usual stable model structure. The equivalence is a composite of two Quillen pairs going in opposite directions:

$$
\text { pro-spectra } \rightarrow \text { ind-spectra }{ }^{o p} \leftarrow \text { spectra }^{o p} .
$$

We have indicated the directions of the left adjoints.

\section{References}

[1] M. Ando and J. Morava, A renormalized Riemann-Roch formula and the Thom isomorphism for the free loop space, in Topology, geometry, and algebra: interactions and new directions (Stanford, CA, 1999), 11-36, Contemp. Math., 279, Amer. Math. Soc., Providence, RI, 2001. MathReview

[2] G. Z. Arone, K. Bauer, J. D. Christensen, and D. C. Isaksen, A classification of polynomial functors using pro-spectra, in preparation.

[3] M. Artin, A. Grothendieck, and J. L. Verdier, Théorie des topos et cohomologie étale des schémas, Lecture Notes in Mathematics, vol. 269, Springer Verlag, 1972. MathReview

[4] M. Artin and B. Mazur, Etale homotopy, Lecture Notes in Mathematics, vol. 100, Springer Verlag, 1969. MathReview

[5] J. C. Becker and D. H. Gottlieb, A history of duality in algebraic topology, History of topology, North-Holland, Amsterdam, 1999, 725-745. MathReview

[6] A. K. Bousfield and E. M. Friedlander, Homotopy theory of $\Gamma$-spaces, spectra, and bisimplicial sets, Geometric Applications of Homotopy Theory, vol. II (Proc. Conf., Evanston, IL, 1977), Lecture Notes in Mathematics, vol. 658, Springer Verlag, 1978, 80-130. MathReview

[7] R. L. Cohen, J. D. S. Jones and G. B. Segal, Floer's infinite dimensional Morse theory and homotopy theory, in The Floer memorial volume, 297-325, Progr. Math., 133, Birkhäuser, Basel, 1995. MathReview 
[8] D. Dugger and D. C. Isaksen, Etale homotopy types and sums-of-squares formulas, in preparation.

[9] W. G. Dwyer and J. Spaliński, Homotopy theories and model categories, Handbook of algebraic topology, North-Holland, 1995, 73-126. MathReview

[10] D. A. Edwards and H. M. Hastings, Cech and Steenrod homotopy theories with applications to geometric topology, Lecture Notes in Mathematics, vol. 542, Springer Verlag, 1976. MathReview

[11] A. D. Elmendorf, I. Kriz, M. A. Mandell, and J. P. May, Rings, modules, and algebras in stable homotopy theory, Mathematical Surveys and Monographs, vol. 47, American Mathematical Society, 1997. MathReview

[12] P. S. Hirschhorn, Model categories and their localizations, Mathematical Surveys and Monographs, vol. 99, American Mathematical Society, 2003. MathReview

[13] M. Hovey, Model categories, Mathematical Surveys and Monographs, vol. 63, American Mathematical Society, 1999. MathReview

[14] M. Hovey, B. Shipley, and J. Smith, Symmetric spectra, J. Amer. Math. Soc. 13 (2000), no. 1, 149-208. MathReview

[15] D. E. Hurtubise, The Floer homotopy type of height functions on complex Grassmann manifolds, Trans. Amer. Math. Soc. 349 (1997), no. 6, 2493-2505. MathReview

[16] D. C. Isaksen, A model structure for the category of pro-simplicial sets, Trans. Amer. Math. Soc. 353 (2001), 2805-2841. MathReview

[17] D. C. Isaksen, Calculating limits and colimits in pro-categories, Fund. Math. 175 (2002), no. 2, 175-194. MathReview

[18] D. C. Isaksen, Etale realization on the $\mathbb{A}^{1}$-homotopy theory of schemes, Adv. Math. 184 (2004), no. 1, 37-63. MathReview

[19] D. C. Isaksen, Strict model structures for pro-categories, in Algebraic Topology: Categorical Decomposition Techniques (Skye, 2001), Progress in Mathematics, vol. 215, Birkhäuser, 2003, 179-198. MathReview

[20] D. C. Isaksen, Completions of pro-spaces, Math. Zeit., to appear.

[21] J. P. May, Stable algebraic topology, 1945-1966, History of topology, NorthHolland, Amsterdam, 1999, 665-723. MathReview

[22] C. V. Meyer, Approximation filtrante de diagrammes finis par Pro-C, Ann. Sci. Math. Québec 4 (1980), no. 1, 35-57. MathReview

[23] D. G. Quillen, Homotopical algebra, Lecture Notes in Mathematics, vol. 43, Springer Verlag, 1967. MathReview

[24] E. H. Spanier and J. H. C. Whitehead, Duality in homotopy theory, Mathematika 2 (1955), 56-80. MathReview

[25] D. Sullivan, Genetics of homotopy theory and the Adams conjecture, Ann. of Math. (2) 100 (1974), 1-79. MathReview

Dept of Math, University of Western Ontario, London, Ontario, Canada

Department of Mathematics, Wayne State University, Detroit, MI 48202, USA

Email: jdc@uwo.ca and isaksen@math.wayne.edu Received: 7 August 2004 